\title{
Características clínicas y demográficas de los pacientes con quiste de colédoco atendidos en el Hospital del Niño Dr. José R. Esquivel en Panamá durante los años 2005 a 2018. \\ Clinical and demographic characteristics of patients with choledochal cysts treated at the Hospital del Niño Dr. José R. Esquivel in Panama during the years 2005 to 2018.
}

\author{
Autores: $\quad$ Dr. José D. Cención 1 \\ Dr. Ricardo A Chanis A. ${ }^{2}$ \\ Dr. Joel Méndez ${ }^{3}$
}

Recibido para publicación: 2 de julio de 2019

Aceptado para publicación: 24 de julio de 2019

\begin{abstract}
Resumen
Introducción: El quiste de colédoco es una condición poco frecuente, se caracteriza por una dilatación de la vía biliar intrahepática y extrahepática. La presentación clínica es variable e incluye principalmente la presencia de masa abdominal, vómitos, ictericia y dolor abdominal,

Materiales y métodos: El objetivo de este estudio es determinar las características clínicas y epidemiológicas de pacientes con quiste de colédoco atendidos en el Hospital del Niño Dr José Renán Esquivel durante los años 2005 a 2018.Se realizó un estudio descriptivo retrospectivo, con interés en las características demográficas como edad, sexo, lugar de procedencia, grupo étnico y las características clínicas como síntomas, método diagnóstico, tipo de quiste reportado, histopatología, y complicaciones.
\end{abstract}

Resultados: La $\mathrm{n}$ fueron 36 pacientes ( $\mathrm{n}=25$ femenino y $\mathrm{n}=11$ masculinos). El grupo de edad de 0-4 años representó el $67 \%$ de los sujetos del estudio. Las principales manifestaciones clínicas fueron dolor abdominal, vómitos e ictericia. El método diagnóstico principal utilizado fue la ecografía abdominal. Principales complicaciones postquirúrgicas colangitis, pancreatitis y sangrado digestivo. La mayoría de los quistes clasificados son Todani IC Y IV.

Conclusiones: El quiste de colédoco es una patología presente en Panamá con una frecuencia de 2.8 casos por año, Palabras clave: quiste de colédoco, ictericia, dolor abdominal, complicaciones, patología.

\footnotetext{
Abstract:

Introduction: Choledochal cysts are a rare condition, characterized by dilatation of the intrahepatic and extrahepatic bile ducts. The clinical presentation is variable and includes the presence of an abdominal mass, jaundice, fever, nausea, vomiting and abdominal pain.

' Pediatra / Residente de Gastroenterología Pediátrica, Hospital del Niño Dr. José R. Esquivel. Panamá, República de Panamá. correo electrónico: danielcencion@hotmail.com

2 Pediatra Gastroenterólogo, Jefe del Servicio de Gastroenterología Pediátrica, Hospital del Niño Dr. José R. Esquivel.

3 Médico Epidemiólogo, Hospital del Niño Dr. José Renán Esquivel. Panamá, República de Panamá.
} 
Materials and methods: The objective of this study is to determine the clinical and epidemiological characteristics of patients with choledochal cyst treated at the Hospital del Niño Dr. José Renán Esquivel during the years 2005 to 2018. A retrospective descriptive study was conducted, with interest in demographic characteristics such as age, sex, place of origin, ethnic group and clinical characteristics such as symptoms, diagnostic method, type of cyst reported, histopathology, complications.

Results: The $\mathrm{N}$ were 36 patients ( $n=25$ female and $n=11$ male). The age group of $0-4$ years represented $67 \%$ of the study subjects. The main clinical manifestations were abdominal pain, vomiting and jaundice. The main diagnostic method used was the abdominal ultrasound. Main postsurgical complications cholangitis, pancreatitis and digestive bleeding. The majority of the classified cysts are Todani IC and IV.

Conclusions: The choledochal cyst is a pathology present in Panama with 2.8 cases per year.

Keywords: choledochal cyst, jaundice, abdominal pain, complications, pathology.

Conflicto de interés: Los autores refieren no tener conflicto de interés.

\section{Introducción}

El quiste de colédoco es una patología de los conductos biliares, que se debe sospechar principalmente ante la presencia de dolor abdominal, ictericia y masa abdominal. Dichos hallazgos se pueden encontrar en la población pediátrica hasta en $85 \%$. Es una condición infrecuente, tienen una incidencia en la población occidental de 1 en 150,000 nacidos vivos por año, aunque se ha informado que puede ser tan alta como 1 en 15,000 nacimientos en Australia y 1 en 13,500 en Estados Unidos, sin embargo en algunas poblaciones asiáticas puede ser tan altas como 1 en 1,000 nacidos vivos. ${ }^{1-5}$

La etiología exacta no se conoce, se ha reportado que entre el 80-95\% de los quistes de colédoco están asociados a una unión anómala pancreática duodenal, donde la unión del conducto biliar común con el pancreático ocurre fuera del duodeno, permitiendo así reflujo de jugo pancreático hacia el árbol biliar. Otras hipótesis sugieren una pared débil del conducto biliar, una presión intraluminal incrementada, anomalías en la inervación autónoma, obstrucción del conducto biliar y disfunción del esfínter de Oddi.2,4

Los quistes de colédoco se asocian con complicaciones tales como estrecheces ductales, formación de cálculos, colangitis, ruptura del quiste, y cirrosis biliar secundaria.
Algunos tipos de quistes de colédoco se han asociado con malignidad.

\section{Material y Método}

Se realizó un estudio descriptivo retrospectivo para evaluar las características clínicas y demográficas de los pacientes atendidos durante los años 2005 a 2018 con diagnóstico de quiste de colédoco.

Se estudiaron variables como edad, sexo, procedencia, grupo étnico, signos y síntomas, método de diagnóstico, tipo de quiste de colédoco, características histológicas de haberse realizado biopsia, complicaciones postoperatorias.

La población de estudio consiste en los expedientes de pacientes con diagnóstico de quiste de colédoco desde enero de 2005 a noviembre de 2018. El tipo de muestreo fue por conveniencia con una n de 36.

Se revisaron los expedientes y se tomaron datos en instrumento de recolección confeccionado por el investigador para consignar la información clínica y demográfica necesaria, de acuerdo a los objetivos del estudio. Para el análisis se utilizaron medidas de tendencia central, se tabularon y realizaron gráficos con programa Excel de Microsoft Office. 


\section{Resultados:}

De los 36 pacientes que conformaban la $n$, se encontró que el $69 \%$ pertenecían al sexo femenino $(n=25)$ y $31 \%$ masculino. $(n=11)$. La edad media calculada por datos agrupados fue 4.2 años, con una desviación estándar de 4.27. ( Gráfica 1).

Gráf. 1. Distribución de acuerdo a la edad de pacientes con diagnóstico de quiste de colédoco atendidos en el Hospital Dr. José R. Esquivel durante los años 2005 al 2018.

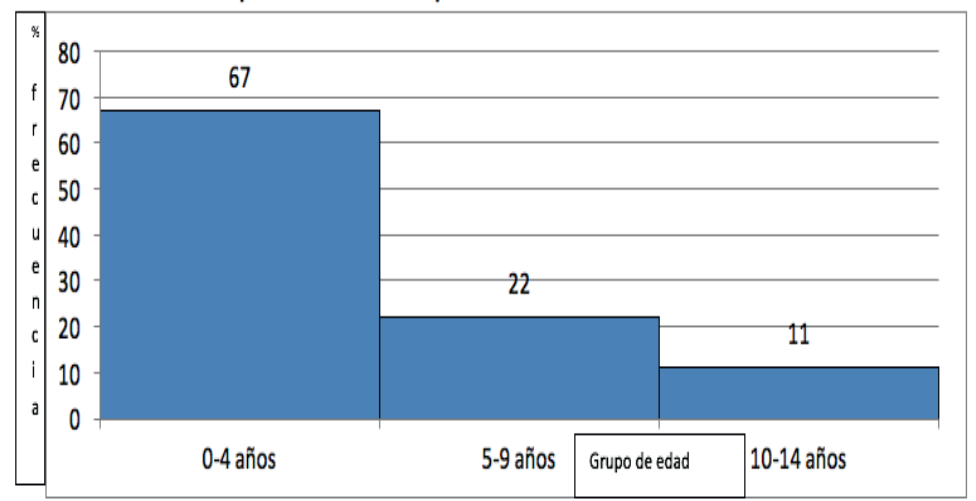

"Fuente: Departamento de Registros médicos Hospital del Niño Dr. José R. Esquivel

Tabla 1.Distribución de acuerdo a procedencia de los pacientes con diagnóstico de quiste de colédoco atendido en Hospital Dr. José R. Esquivel durante los años 2005 al 2018.

\begin{tabular}{|lcc|}
\hline Provincia & Frecuencia & Porcentaje \% \\
\hline Bocas del Toro & 0 & 0 \\
\hline Coclé & 1 & 3 \\
Colón & 3 & 8 \\
Chiriquí & 0 & 0 \\
Darién & 0 & 0 \\
\hline Herrera & 2 & 6 \\
\hline Los Santos & 3 & 8 \\
\hline Panamá & 10 & 28 \\
\hline Panamá Oeste & 12 & 33 \\
\hline Veraguas & 3 & 8 \\
\hline Comarca Kuna Yala & 2 & 6 \\
\hline Otras comarcas & 0 & 0 \\
\hline Total & 36 & 100 \\
\hline
\end{tabular}

*Fuente: Departamento de Registros médicos Hospital del Niño Dr José R. Esquivel

En este estudio se encontró el diagnóstico de quiste de colédoco en $78 \%$ de pacientes mestizos ( $n=28)$, en el grupo negro e indígena el mismo porcentaje $8 \%(n=3)$ y en el grupo asiático $6 \%(n=2)$.
Tabla 2. Descripción de las principales manifestaciones clínicas según grupo de edad de los pacientes con diagnóstico de quiste de colédoco atendido en Hospital Dr. José R. Esquivel durante los años 2005 al 2018.

\begin{tabular}{|lcccccc|}
\hline Grupo de edad & $\begin{array}{c}\text { Dolor } \\
\text { abdominal }\end{array}$ & Vómitos & Ictericia & Fiebre & $\begin{array}{c}\text { Masa } \\
\text { palpable }\end{array}$ & $\begin{array}{c}\text { Abdomen } \\
\text { agudo }\end{array}$ \\
\hline 0-1año & 2 & 5 & 11 & 5 & 2 & 2 \\
\hline 2-3años & 3 & 3 & 1 & 1 & 1 & 0 \\
4-5años & 6 & 5 & 2 & 1 & 0 & 0 \\
6-7años & 2 & 2 & 1 & 1 & 0 & 0 \\
8-9ños & 3 & 2 & 0 & 0 & 0 & 0 \\
10-11años & 1 & 1 & 0 & 0 & 0 & 0 \\
12-13años & 2 & 2 & 0 & 0 & 0 & 0 \\
14-15años & 1 & 0 & 0 & 0 & 0 & 0 \\
Total & 20 & 20 & 15 & 8 & 3 & 2 \\
\hline
\end{tabular}

Nota: el universo está compuesto por 36 pacientes, los pacientes podian presentar más de 1 manifestación clínica. "Fuente: Departamento de Registros médicos Hospital del Niño Dr José R. Esquivel.

Tabla 3.Descripción de los estudios para hacer el diagnóstico de pacientes con quiste de colédoco atendidos en Hospital Dr. José R. Esquivel durante los años 2005 al 2018.

\begin{tabular}{|l|ccc|}
\hline Estudio & Frecuencia & Total & Porcentaje \% \\
\hline Ecografia & 34 & 36 & 94 \\
\hline $\begin{array}{l}\text { Resonancia } \\
\text { magnética }\end{array}$ & 15 & 36 & 42 \\
$\begin{array}{l}\text { Tomografia } \\
\text { computarizada }\end{array}$ & 4 & 36 & 11 \\
\hline Cirugía & 3 & 36 & 8.3 \\
\hline CPRE & 2 & 36 & 6 \\
\hline
\end{tabular}

Nota: el universo está compuesto por 36 pacientes, los pacientes se les realizó el diagnóstico con 10 más estudio.`Fuente: Departamento de Registros médicos Hospital del Niño Dr. José R. Esquivel

Tabla 4.Descripción de los hallazgos de laboratorios en pacientes con quiste de colédoco atendidos en Hospital Dr. José R. Esquivel durante los años 2005 al 2018.

\begin{tabular}{llc|}
\hline Hallazgo de laboratorio & Frecuencia & Porcentaje \% \\
& $\mathbf{S i}$ & \\
\hline Hipertransaminemia & 21 & 58 \\
Anemia & 14 & 38 \\
Leucocitosis & 13 & 36 \\
Hiperbilirrubinemia directa & 11 & 30 \\
\hline Amilasa y lipasa elevadas & 6 & 17 \\
\hline Prolongación de tiempos de coagulación & 4 & 11 \\
\hline
\end{tabular}
"Fuente: Departamento de Registros médicos Hospital del Niño Dr. José R. Esquivel

Nota: el universo está compuesto por 36 pacientes.

Tabla 5. Tipo de quiste de colédoco según clasificación de Todani diagnosticado en pacientes atendidos en Hospital Dr. José R. Esquivel durante los años 2005 al 2018

\begin{tabular}{|ccc|}
\hline Clasificación de Todani & Frecuencia & Porcentaje \\
\hline IA & 3 & 14 \\
IB & 1 & 5 \\
IC & 9 & 43 \\
II & 2 & 9 \\
III & 1 & 5 \\
IV & 4 & 19 \\
V & 1 & 5 \\
Total & 21 & 100 \\
\hline *Fuente: Departamento de Registros médicos Hospital del Niño Dr. José R. Esquivel
\end{tabular}


De los 21 expedientes clínicos donde se consignaba la clasificación de quiste de colédoco la mayoría correspondía a quistes IC.

Tabla 6.Caracteristicas histológicas de pacientes con quiste de colédoco atendidos en Hospital Dr. José R. Esquivel durante los años 2005 al 2018.

\begin{tabular}{|lcc|}
\hline Hallazgo & Frecuencia & Porcentaje \% \\
\hline Fibrosis & 5 & 21 \\
\hline Sin alteraciones & 5 & 21 \\
\hline Cirrosis & 6 & 25 \\
\hline Infiltrado inflamatorio crónico & 8 & 33 \\
\hline
\end{tabular}

De los 36 expedientes revisados, se consignó reporte histológico en 24 de ellos. El resto no consignado obedecía a que no se tomó biopsia al realizar la cirugía, o en menor cantidad en pacientes que solicitaron ser operados en otra institución.

Tabla 7.Complicaciones postquirúrgicas en los pacientes con quiste de colédoco atendidos en Hospital Dr. José R. Esquivel durante los años 2005 al 2018.

\begin{tabular}{|lcc|}
\hline \multicolumn{1}{|c|}{ Complicaciones } & Frecuencia & Porcentaje (\%) \\
\hline Ninguna & 15 & 52 \\
\hline Colangitis & 4 & 14 \\
Pancreatitis & 4 & 14 \\
Sangrado digestivo & 4 & 14 \\
Obstrucción de anastomosis & 2 & 7 \\
\hline Insuficiencia hepática & 1 & 3 \\
Hipertensión portal & 1 & 3 \\
\hline Encefalopatia bilirrubínica & 1 & 3 \\
Peritonitis & 1 & 3 \\
\hline
\end{tabular}

"Fuente: Departamento de Registros médicos Hospital del Niño Dr. José R. Esquivel

Del total de 36 pacientes, 29 recibieron intervención quirúrgica de los cuales se consignan las complicaciones posteriores. 5 pacientes no se consignan si hubo o no complicaciones postoperatorias, ya que decidieron operarse en otra institución y no continuaron seguimiento en el hospital. Los pacientes podían presentar 1 o más complicaciones relacionadas al quiste de colédoco. En el estudio se encontró 2 muertes dentro de los pacientes como desenlace de su patología, antes de poder ser operados o trasplantados lo que corresponde a una tasa de letalidad de 5.5\%.

Tabla 8.Período de tiempo entre la realización del diagnóstico y el tratamiento quirúrgico en pacientes con quiste de colédoco atendidos en Hospital del niño Dr. José Renán Esquivel durante 2005 a 2018

\begin{tabular}{|ccc|}
\hline $\begin{array}{c}\text { Rango de tiempo } \\
\text { (Meses) }\end{array}$ & Frecuencia & Porcentaje \% \\
\hline $0-11$ & 24 & 83 \\
\hline $12-23$ & 2 & 7 \\
\hline $24-35$ & 1 & 3 \\
\hline $36-47$ & 0 & 0 \\
\hline $48-59$ & 1 & 3 \\
\hline $60-71$ & 1 & 3 \\
\hline
\end{tabular}

*Fuente: Departamento de Registros médicos Hospital del Niño Dr. José R. Esquivel
De los 36 pacientes estudiados, 29 recibieron tratamiento quirúrgico en la institución que consistía en resección quirúrgica abierta del quiste y realización de anastomosis hepático-yeyunal en $Y$ de Roux, en base a éstos, colocamos el período de tiempo desde que se realizó el diagnóstico hasta que recibieron la intervención quirúrgica. Se reporta una media del período de tiempo entre el diagnóstico y la cirugía de 11.4 meses, con una desviación estándar de 79.9 .

Tabla 9.Características histológicas según tiempo esperado para tratamiento quirúrgico en pacientes con quiste de colédoco atendidos en Hospital Dr. José R. Esquivel durante los años 2005 a 2018.

\begin{tabular}{ccccc}
\hline $\begin{array}{c}\text { Rango de } \\
\text { tiempo } \\
\text { (Meses) }\end{array}$ & Fibrosis & Sin alteraciones & Cirrosis & $\begin{array}{c}\text { Infiltrado } \\
\text { inflamatorio } \\
\text { crónico }\end{array}$ \\
\hline $0-11$ & 5 & 2 & 6 & 6 \\
\hline $12-23$ & 0 & 2 & 0 & 0 \\
\hline $24-35$ & 0 & 0 & 0 & 1 \\
\hline $36-47$ & 0 & 0 & 0 & 0 \\
$48-59$ & 0 & 1 & 0 & 0 \\
\hline $60-71$ & 0 & 0 & 0 & 1 \\
\hline Total & 5 & 5 & 6 & 8 \\
\hline
\end{tabular}

Fuente: Departamento de Registros médicos Hospital del Niño Dr. José R. Esquivel

\section{Discusión}

En un periodo de 13 años fueron diagnosticados 36 pacientes por quiste de colédoco atendidos en nuestra institución. El 69\% de los casos correspondió al sexo femenino. Los quistes de colédoco son una condición poco conocida, su incidencia en las poblaciones occidentales ha sido estimada de 1:100,000 a 1:150,000.1,2,4,5,6

Se presentan más comúnmente en mujeres que en hombres 3:1 a 4:1, y la mayoría de los diagnósticos se realizaban en la edad pediátrica. ${ }^{3,5,6}$ Chanis $^{7}$ en estudio previo encontró igual resultado donde el sexo femenino predominó, al igual que Palmer ${ }^{8}$ en revisión de casos en Tabasco, México que el $72 \%$ correspondió a niñas. Mientras en China, Liu-Bin Shi de 108 pacientes , 85 eran del sexo femenino. ${ }^{10}$

En nuestro estudio el $67 \%$ de los pacientes fueron diagnosticados entre los 0 - 4 años de edad. Esto se corresponde con López ${ }^{11}$ en Valencia, España quien revisó un periodo de 20 años donde el diagnóstico se realizó entre 1 a 4 años de edad, igual que Liras ${ }^{12}$ en cuya serie el periodo fue entre los 8 meses a 4 años. 
Panamá es considerado históricamente un país de tránsito donde convergen diferentes grupos étnicos, algunos que han permanecido en el istmo permitiendo el mestizaje y asentándose en diferentes lugares del país. Desde 1850 Panamá ha recibido inmigrantes de origen asiático (principalmente chinos). En este estudio se encontró el diagnóstico de quiste de colédoco en 78\% de pacientes mestizos, en el grupo negro e indígena el mismo porcentaje $8 \%$ y en el grupo asiático $6 \%$.

Jimenez ${ }^{14}$ documentó la frecuencia comparativa de casos por año en diferentes países entre ellos Panamá ( 2.40 casos/año), en nuestro estudio se encontró una frecuencia de 2.8 casos por año. Las manifestaciones clínicas principales fueron: dolor abdominal, vómitos, ictericia, fiebre, masa palpable y abdomen agudo, aunque al momento de su diagnóstico podían presentar más de síntoma o signo clínico. Esto se corrobora con lo documentado por Soares ${ }^{15}$, Palmer ${ }^{8}$ que encontraron el dolor abdominal, masa palpable, vómitos e ictericia en orden de frecuencia como manifestaciones principales. Chanis encontró 50\% de los pacientes cursaban con dolor abdominal e ictericia como principales manifestaciones?

En el 94\% de nuestros casos el diagnóstico se hizo por ecografía abdominal, como lo reportado por Jiménez ${ }^{14}$, Medel ${ }^{16}$ en México refieren el uso de ultrasonido abdominal principalmente, con una sensibilidad del estudio de 97\%, aunque señalan este no permite clasificar el quiste y detalles de la anatomía. y Vivas ${ }^{17}$ en España, encontró que el 94\% de los casos fue diagnosticado por ecografía. En el 54\% de los casos hubo elevación de las transaminasas seguido de leucocitosis, y anemia, estos hallazgos se corresponden a los encontrados por Jiménez ${ }^{14}$ donde todos cursaron con elevación de pruebas de funcionamiento hepático y solo un caso presentó elevación de amilasa sérica.

De los 36 casos encontrados solo a 21 se le realizó la clasificación de Todani, de estos 43\% correspondió al grupo IC, seguido del grupo IV esto concuerda con la literatura que refiere que los quistes de colédoco tipo I son los más frecuentes con porcentaje de 50-85\%, seguidos en frecuencia por tipo IV 15-35\%, lo único que difiere que el tipo $V$ puede presentarse hasta en $20 \%$, lo que no observamos en nuestra serie $1.8,12,14$ Fue consignado el reporte histopatológico en 24 sujetos, en el 33\% de estos había infiltrado inflamatorio crónico, un $25 \%$ con cirrosis, $21 \%$ sin alteraciones patológicas y fibrosis sin especificar grado, respectivamente. En los pacientes con intervención quirúrgica tardía el diagnóstico de cirrosis fue más frecuente. En estudio previo realizado por Chanis encontró en 80\% de los casos pericolangitis y $20 \%$ con fibrosis.?
En el 52\% de los casos no hubo complicación post-quirúrgica, pero la pancreatitis, colangitis y sangrado digestivo fueron las principales como se mostró en la tabla 7.

Existen complicaciones inherentes al quiste y a su tratamiento, entre las que se señalan la colangitis ascendente, pancreatitis, cirrosis biliar, hipertensión portal y transformación maligna. Se describe la aparición de úlceras pépticas en cualquier tipo de hepatoenterostomía ${ }^{18}$. Vivas ${ }^{17}$ refiere complicaciones relacionadas al diagnóstico tardío, que ellos consideran mayor a 2 años, como colangitis, dehiscencia de la anastomosis, sepsis, falla renal aguda, hipertensión arterial. Dentro del grupo estudiado se reportó 2 fallecidos como desenlace de la patología antes de ser operados, con una tasa de letalidad de 5.5\%.

En el estudio se reporta una media de tiempo entre el que se realiza el diagnóstico y se realizó la cirugía de 11.4 meses. Esta prolongación de tiempo para el manejo definitivo puede obedecer al diagnóstico tardío, perdida del seguimiento en la consulta externa, mora quirúrgica, pero esto no fue considerado en nuestro estudio.

El tratamiento del quiste de colédoco es quirúrgico, el objetivo es la resección total y el mantenimiento de un flujo biliar apropiado. El tiempo para la realización de la cirugía luego del diagnóstico debe ser breve, en pacientes asintomáticos refieren no más de 6 meses y 2 meses en pacientes sintomáticos. En lactantes captados fuera del período neonatal se recomienda la resección en el tiempo más breve posible, independiente de la edad, por la pronta progresión a daño hepático. 19,20,21,22,23, Bobadilla ${ }^{24}$ en el Centro médico nacional siglo XXI en México de 2006 a 2012, los pacientes fueron sometidos a hepato-yeyuno anastomosis en $Y$ de Roux que es el tratamiento quirúrgico recomendado en la literatura. El procedimiento quirúrgico se realizó dentro de la primera semana luego del diagnóstico.

La literatura refiere que de instaurarse el tratamiento de forma precoz el resultado generalmente es favorable, sin embargo, se debe recordar que una de las complicaciones más temidas es la malignización del tejido presentándose como colangiocarcinoma. ${ }^{25,26,27,28,29}$ 


\section{Conclusiones}

En Panamá, el quiste de colédoco se presenta con una frecuencia de 2.8 casos por año, predomina en el sexo femenino, la edad media del diagnóstico es 4.2 años. Los grupos más frecuentes fueron los IC, IA y tipo IV según la clasificación de Todani para quiste de colédoco.

El seguimiento del paciente con quiste de colédoco debe ser de por vida, dado el riesgo de malignidad en esta patología. Se sugiere revisión mensual los primeros 6 meses y posteriormente semestral, esto debe ser individualizado, teniendo en cuenta que quistes de colédoco tipo I y IV son más propensos a malignización.

\section{Limitaciones}

La falta de consignación de la información en el expediente y poca asistencia a citas postoperatoria de algunos de los sujetos fueron algunas de las limitaciones en el estudio. Dificultades para obtención o prolongación en el tiempo para la intervención fue una de las causas para que algunos sujetos del estudio decidieran operarse en otra institución, dificultando así la obtención de datos como complicaciones postoperatorias y el informe de patología. En algunos pacientes operados por quiste de colédoco no se les realizó biopsia o no se envió pieza anatómica para análisis por patología.

\section{Referencias.}

1. Topazian M, Chopra S, Rand EB, Grover S. Biliary Cyst url: https://www.uptodate.com/contents/biliary-cysts/print?sour ce $=$ search_result\&search $=$ choledochal\%20cyst\%20children\& selectedTitle $=1 \sim 84$

2. Sánchez J, Gómez S, Morales C, Hoyos SI. Quistes del colédoco. Rev Colomb Cir. 2015;30:296-305.

3. Ruíz Cubillo M, Brenes Valverde D, Ayi Wong J. Quiste de colédoco. Rev Méd Cos Cen 2011: 68 (596) ;49-56.

4. Lipsett PA, Pitt HA, Colombani PM, Boitnott JK, Cameron JL. Choledochal cyst disease. A changing pattern of presentation. Ann Surg 1994; 220 (5):644- 52.

5. O'Neill JA Jr, Choledochal cyst. Curr Probl Surg 1992; 29 (2):361-340.

6. Carneros Martín JA, García Durán F, Tomás Moros E, Villa Poza JC, Rodríguez Agulló JL. Quistes de colédoco. Revista de la ACAD 2009; 25 (4):227-231

7. Chanis R, Norte G, Troitiño M, Forero I. Quiste de colédoco: evolución,evaluación, y tratamiento. Rev Hospital del Niño. 2005; 21 (2): 174-178.
8. Palmer-Becerra JD, Ulloa P. Tratamiento de los quiste de colédoco en la edad pediátrica. Un revisón de 24 años. Acta Pediatr Mex 2010;31(1):11-15.

9. Tam J. Huellas China en Panama: 150 años de presencia. $1^{a}$ ed. Único Impresores. ISBN 9962-02-444-7. 2006.

10. Shi LB, Peng SY, Meng XK et al. Diagnosis and treatment of congenital choledochal cyst: 20 years' experience in China. World J Gastroenterol 2001;7(5):732-734

11. López Ruíz R, Aguilera Alonso D, Múñoz Aguilar G, Fonseca Martín R. Quistes de colédoco, una causa inusual de ictericia en pediatría. Rev Chil Pediatr. 2016;87(2):137-142. doi: 10.1016/j.rchipe.2015.08.009. Epub 2015 Oct 23

12. Liras Muñoz J. Bueno Recio J. Sánchez Abuín A et al. Tratamiento del quiste de colédoco: ¿Cirugía abierta o endoscópica? Cir Pediatr 2005; 18 (2): 73-76

13. Contraloria general de la República de Panamá, Instituto Nacional de estadística y censo. Panamá en cifras años 2010-2014.

14. Jiménez y Felipe JH. Malformaciones de las vías biliares en pediatría.Quiste de colédoco. Revista Mexicana de Cirugía Pediátrica. 2010; 17 (1): 11-18.

15. Soares KC, Arnaoutakis DJ, Kamel I et al. Choledochal cysts: presentation, clinical differentiation, and management. J Am Coll Surg. 2014; 219 (6): 1167-80. doi:10.1016/j.jamcollsurg. 2014.04.023. Epub 2014 Jun 27.

16. Medel-Morales IB, Pérez-Hernández BM, Ortíz-Galván RC, Fernando-Oliver E. Experiencia quirúrgica en el manejo de pacientes con quiste de colédoco, análisis de 10 años. Rev. MEx. Cir Ped. 2015 ;19 (1): 10-18.

17. Vivas-Colmenares GV, Millán-López A, De Agustín JC. Optimización en la estrategia diagnóstica y terapéutica en el quiste de colédoco. Rev Chil Cir. 2015; 67(3) :278-284.

18. Yamataka A. Ohshiro K, Okada Y et al . Complication after cyst excision with hepaticoenterostomy for choledochal cyst and their surgical management in children versus adults. J Pediatr Surg 1997;32(7):1097-102

19. Suita S, Shono K, Kinugasa Y, Kubota M, Matsuo S. Influence of age on the presentation and outcome of choledochal cyst. J Pediatr Surg 1999; 34(12): 1765-8.

20. Astudillo P, Vasconez F, Zambrano E. Nuevos aportes en el diagnóstico y tratamiento del quiste de colédoco. Rev Ecuat Pediatr .2006; 7(2): 5-10.

21. Metcalfe MS, Wemyss-Holden SA, Maddern GJ. Management dilemmas with choledochal cysts. Arch Surg 2003; 138(3): 333-9.

22. Acevedo D, Rivas IA, Rodríguez A. Quiste de colédoco, cuadro clínico diagnóstico y tratamiento. Acta Médica Grupo Ángeles. 2015;13 ( 3):171-176. 
23. Galindo F. Dilataciones quísticas congénitas y divertículos de vías biliares. F.Galindo: Enciclopedia Cirugía Digestiva. Capítulo IC 2016; tomo IV-439: pág. 1- 20. http://www.sacd.org. ar/ctreintaynueve.pdf

24. Fernández Bobadilla N, Flores Calderón J, Bernabé García M, Lagarda Cuevas J. Caracterización clínica de niños con quiste de colédoco. An Med (Mex) 2016;61 (1): 11-19.

25. Cohen A, Corrales J. Otero H, Arrea C. Quiste congénito del colédoco. Rev.Méd.Hosp.Nal.Niños Costa Rica 1983;18(2); 223-232.

26. Zheng X, Gu W, Xia H, et al. Surgical treatment of type IV-A choledochal cyst in a single institution: children vs. adults. J Pediatr Surg 2013; 48(10):2061-6.

27. Lee SE, Jang JY, Lee YJ et al. Choledochal cyst and associated malignant tumors in adults: a multicenter survey in South Korea. Arch Surg 2011; 146 (10):1178-84. doi: 10.100/ archsurg.2011.243.

28. Jabłońska B. Biliary cysts: etiology, diagnosis and management. World J Gastroenterol 2012; 18 (35):4801-10.

29. Saing H, Han H, Chan KL et al. Early and late results of excision of choledochal cysts. J Pediatr Surg 1997; 32 (11):1563-6. 